\title{
Operative Fluoroscopic Correction Is Reliable and Correlates With Postoperative Radiographic Correction in Periacetabular Osteotomy
}

\author{
James D. Wylie MD, MHS, Jeremy A. Ross MD, Jill A. Erickson PA-C, \\ Mike B. Anderson MSc, Christopher L. Peters MD
}

Published online: 12 September 2016

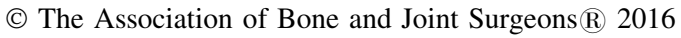

\begin{abstract}
Background Intraoperative fluoroscopy is commonly used to both guide the osteotomy and judge correction of the acetabular fragment in periacetabular osteotomy (PAO). Prior studies that have compared intraoperative fluoroscopic correction with postoperative radiographic correction were small studies that did not report intra- or interreader reliability.

Questions/purposes (1) What is the correlation between intraoperative fluoroscopic correction in PAO compared with the correction seen on postoperative radiographs? (2) What is the reliability of radiographic measures of correction in $\mathrm{PAO}$ ?

Methods We performed a retrospective study of 121 patients (141 hips) who underwent PAO for symptomatic hip dysplasia at a tertiary referral center. Patients were included in the study if they had preoperative radiographs, intraoperative fluoroscopy, and minimum 6-week postoperative radiographs. Of the 272 PAO procedures performed in this time period, 61
\end{abstract}

One of the authors certifies that he (CLP), or a member of his immediate family, has or may receive payments or benefits, during the study period, an amount of USD 100,001 to USD 1,000,000 from Biomet, Inc (Warsaw, IN, USA).

All ICMJE Conflict of Interest Forms for authors and Clinical Orthopaedics and Related Research ${ }^{\circledR}$ editors and board members are on file with the publication and can be viewed on request.

Each author certifies that his or her institution approved or waived approval for the human protocol for this investigation and that all investigations were conducted in conformity with ethical principles of research.

J. D. Wylie, J. A. Ross, J. A. Erickson, M. B. Anderson,

C. L. Peters $(\square)$

Department of Orthopaedic Surgery, University of Utah, 590

Wakara Way, Salt Lake City, UT 84108, USA

e-mail: chris.peters@hsc.utah.edu patients who underwent PAO for retroversion and five patients with a history of Perthes disease were excluded as a result of the inability for these radiographic measures to judge fragment correction in PAOs for retroversion and the difficulty in measurement in post-Perthes deformity. Of the 206 PAOs performed for symptomatic acetabular dysplasia, 65 (32\%) could not be analyzed because they lacked appropriate preoperative films, leaving 141 PAOs in 121 patients for analysis. The patients lacking appropriate preoperative films had them performed at an outside facility or had plain films that have since been destroyed. The lateral center-edge angle (LCEA) and acetabular index (AI) on the fluoroscopic views and postoperative radiographs were measured by two authors. The concordance between the amount of correction on intraoperative fluoroscopy and minimum 6-week postoperative measurements was analyzed using the concordance correlation coefficient $\left(r_{c}\right)$ and a Bland-Altman analysis. Intra- and interrater reliability was calculated between measurements.

Results The amount of intraoperative correction of LCEA as measured on fluoroscopic images demonstrated substantial agreement with postoperative radiographs $\left(r_{c}=\right.$ $0.79 ; 95 \%$ confidence interval [CI], 0.73-0.85; $\mathrm{p}<0.001$ ) as did the $\mathrm{AI}\left(\mathrm{r}_{\mathrm{c}}=0.77 ; 95 \% \mathrm{CI}, 0.70-0.84 ; \mathrm{p}<0.001\right)$. The mean difference between intraoperative correction was only $-0.38^{\circ}\left(\mathrm{SD} 3.6^{\circ}\right)$ for LCEA and $-0.84^{\circ}\left(\mathrm{SD} 3.4^{\circ}\right)$ for AI. Interrater reliability for both LCEA and AI also demonstrated substantial agreement $\left(\right.$ all, $\left.r_{c}=0.70-0.90\right)$ for preoperative, operative, and postoperative imaging. Furthermore, intrarater reliability for both LCEA and AI demonstrated almost perfect agreement for all measures (all, $\mathrm{r}_{\mathrm{c}}>0.81$ ).

Conclusions Intraoperative fluoroscopy is an accurate and reliable measure of correction of lateral coverage of the acetabular fragment during PAO. Further studies on measures of anterior coverage and acetabular version are 
needed to validate intraoperative fluoroscopic correction in these planes.

Level of Evidence Level III, diagnostic study.

\section{Introduction}

Periacetabular osteotomy (PAO) is frequently the surgical treatment of choice for symptomatic hip dysplasia in skeletally mature patients [5]. PAO strives to increase the anterior and lateral coverage of the femoral head with the goal of improved pain and function by normalizing the mechanics of the hip after acetabular reorientation [17]. Intraoperatively, fluoroscopy is often used to guide the osteotomy and judge correction of the acetabular fragment. Some have advocated for plain radiographs to be performed in the operating room as a result of the belief that these would more accurately judge fragment reorientation [7].

However, there has been limited investigation on the agreement between the correction seen on intraoperative fluoroscopic images compared with postoperative radiographs. Kühnel et al. [8] reviewed 22 PAOs and found no difference between measurements made on intraoperative radiography and fluoroscopy with the small numbers available; given that fluoroscopy was associated with lower radiation doses, they recommended its use. Lehmann et al. [10] reviewed 50 PAOs and reported the correlation between intraoperative fluoroscopic images and postoperative radiographs. They found that the lateral center-edge angle (LCEA), anterior center-edge angle (ACEA), and the acetabular index (AI) showed the greatest correlation. Although these studies suggest intraoperative fluoroscopy is a useful tool to judge correction in PAO, both studies had a small number of subjects and neither reported the intraor interrater reliability of these measures.

The purpose of this study therefore was to determine the agreement between intraoperative fluoroscopic correction during PAO and the correction seen on postoperative radiographs after PAO. To assess this, we asked two questions: (1) What is the correlation between intraoperative fluoroscopic correction in PAO compared with the correction seen on postoperative radiographs? (2) What is the reliability of fluoroscopic and radiographic measures of correction in PAO?

\section{Patients and Methods}

After receiving a waiver from the institutional review board, we performed a retrospective study of all patients who underwent PAO from November 1, 2001, to June 21, 2015 , at a single tertiary referral center. All procedures were performed by a single surgeon (CLP) for symptomatic acetabular dysplasia. Patients were included in the study if they had preoperative radiographs including a standing AP of the pelvis, intraoperative fluoroscopy with an AP image of the hip, and a minimum of 6-week postoperative AP radiograph of the pelvis in our digital imaging system. Of the 272 PAO procedures performed in this time period, 61 patients who underwent PAO for retroversion and five patients with a history of Perthes disease were excluded as a result of the inability for these radiographic measures to judge fragment correction in PAOs for retroversion and the difficulty in measurement in post-Perthes deformity.

Of the 206 PAOs performed for symptomatic acetabular dysplasia, 65 (32\%) could not be analyzed because they lacked appropriate preoperative films, leaving 141 PAOs in 121 patients for analysis. The patients lacking appropriate preoperative films had these performed at an outside facility or had plain films that have since been destroyed. The mean age was 27 years (range, 14-47 years). The majority of patients were female (79\% [112 of 141]) and the mean body mass index was $24.6 \mathrm{~kg} / \mathrm{m}^{2}$ (range, 17-39 $\left.\mathrm{kg} / \mathrm{m}^{2}\right)$.

\section{Radiographic and Fluoroscopic Measures}

Radiographs were obtained with weightbearing using standardized protocols at both the preoperative and postoperative visits [4]. Intraoperative fluoroscopy images were obtained by first centering the fluoroscope over the pubic symphysis. The fluoroscope was just above the patient so that the obturator foramen and the pelvic ring were visualized. Pelvic tilt and rotation were adjusted to recreate the image of the obturator foramen and pelvic ring seen on the patient's preoperative standing radiograph before taking each image of the hip. The image was considered adequate if the obturator foramina were symmetric and the coccyx was in line with the pubic symphysis. The fluoroscope was then moved over the operative hip, given that the hip was not fully visualized when the beam was centered over the symphysis, and an AP image of the hip was obtained. This allowed evaluation of most of the hemipelvis including the proximal femur.

Each patient included in the study had the LCEA and AI measured on the AP view of preoperative radiographs, posteroanterior view of intraoperative fluoroscopic images, and AP view of postoperative radiographs by two authors who were not involved in the surgical care of these patients (JDW, JAR). One reader was a chief resident with special training in radiographic measures of the hip (JDW), and the other reader was an adult reconstruction fellow who was similarly trained (JAR). All measurements were made digitally in our picture 
Table 1. Concordance and agreement between intraoperative correction on fluoroscopy and postoperative radiographs

\begin{tabular}{llllll}
\hline Radiographic measurement & $\mathrm{r}_{\mathrm{c}}$ & $95 \% \mathrm{CI}$ & Bland-Altman average difference (SD) & $95 \%$ limits of agreement & Pearson's $\mathrm{r}$ \\
\hline LCEA correction & 0.79 & $0.73-0.85$ & $-0.38(3.6)$ & -7.4 to 6.6 & 0.80 \\
AI correction & 0.77 & $0.70-0.84$ & $-0.84(3.4)$ & -7.6 to 5.9 & 0.78 \\
\hline
\end{tabular}

$\mathrm{CI}=$ confidence interval; LCEA = lateral center-edge angle; $\mathrm{AI}=$ acetabular index (Tönnis angle).

archiving and communications (PACS) electronic imaging system. The LCEA was measured as the angle made by a vertical line through the center of the femoral head and a line drawn from the center of the femoral head through the lateral aspect of the acetabular roof (sourcil) [19]. The AI or Tönnis angle was measured as the angle between a line horizontal to the pelvis and a line connecting the medial and lateral aspects of the acetabular roof or sourcil [18]. On all radiographic imaging, a line intersecting the same bony landmark on either side of the pelvis (ie, the inferior aspect of the acetabular teardrop or the inferior aspect of the ischium) was used to orient the measures to the bony pelvis. On the fluoroscopic images, the pelvis was assumed to be horizontal to the fluoroscopic image that was obtained resulting from the standard acquisition protocol explained previously. One month after his initial review of the images, one reader (JAR) reread all images included in the study to determine intraobserver reliability. Radiographic measurements were averaged between the first reads of both observers to determine the correction in patients.

\section{Statistics}

The primary outcomes, agreement between the amount of correction on intraoperative fluoroscopy (intraoperativepreoperative) and 6-week postoperative measurements (postoperative-preoperative), were analyzed using the concordance correlation coefficient $\left(r_{c}\right)$ and a Bland-Altman analysis $[1,11]$. Similarly, $r_{c}$ and a Bland-Altman analysis were used to assess both interrater and intrarater reliability and agreement. Given the inherent limitations of the $r_{c}$, similar to that of the intraclass correlation coefficient, the Bland-Altman analysis is used to describe the clinical relevance of the findings [2, 13]. It should be noted that an $r_{c}$ near one indicates near perfect agreement; however, for ease of interpretation, the $r_{c}$ was interpreted as described by the guidelines of Landis and Koch [9]. Furthermore, we stress the importance of the $95 \%$ limits of agreement (LA) as estimates including some sampling error and should be interpreted as such [2]. Significance was assessed at the 0.05 level. Statistical analysis was performed using Stata Version 14.1 (College Station, TX, USA).

\section{Results}

The amount of intraoperative correction of LCEA as measured on fluoroscopic images demonstrated substantial agreement with postoperative radiographs $\left(r_{c}=0.79 ; 95 \%\right.$ confidence interval [CI], 0.73-0.85; $<<0.001)$ as did the AI correction $\left(r_{c}=0.77 ; 95 \%\right.$ CI, $\left.0.70-0.84 ; p<0.001\right)$ (Table 1). Furthermore, the agreement between the measurements demonstrated a mean difference of $-0.38^{\circ}$ (SD $3.6^{\circ}$ ) for LCEA (Fig. 1) and $-0.84^{\circ}$ (SD 3.4 ${ }^{\circ}$ ) for AI (Fig. 2) demonstrating a minimal difference between the two separate measurements. Furthermore, the $95 \%$ LA are within an acceptable range clinically between the intraoperative fluoroscopy images and the postoperative films for both measurements, suggesting the use of intraoperative fluoroscopic images may be sufficient for assessing acetabular orientation with these measurements. The mean correction for LCEA on intraoperative fluoroscopy was $18^{\circ}$ (range, $6^{\circ}$ $41^{\circ}$ ) and the mean correction for LCEA on postoperative films was $19^{\circ}$ (range, $7^{\circ}-33^{\circ}$; Table 2 ). The mean correction for $\mathrm{AI}$ on intraoperative fluoroscopy was $-17^{\circ}$ (range, $-33^{\circ}$ to $-6^{\circ}$ ) and the mean correction for $\mathrm{AI}$ on postoperative films was $-16^{\circ}$ (range, $-32^{\circ}$ to $-5^{\circ}$; Table 2 ).

Inter- and Intrarater Reliability of Measures of Acetabular Reorientation

Interrater reliability for both LCEA and AI demonstrated substantial agreement (all, $r_{c} \geq 0.70$ ) for preoperative, operative, and postoperative imaging (Table 3 ). The mean difference for interrater agreement was also small, being less than $1.6^{\circ}$, for all measures (Table 3 ). These mean differences are within a clinically acceptable range of difference between measurements of the two observers. The 95\% LA demonstrate that $95 \%$ falls within $5^{\circ}$ to $10^{\circ}$ of the other observer, varying depending on the measure (Table 3 ).

Intrarater reliability for both LCEA and AI also demonstrated almost perfect agreement for all measures. Additionally, the mean differences for preoperative, operative, and postoperative imaging were rather small with all mean differences in a clinically acceptable range, falling within $1.5^{\circ}$ of the prior reading (Table 4). The 95\% LA were similar to the variation between observers with most differences between the repeated measures being within $5^{\circ}$ to $10^{\circ}$ (Table 4 ). 
Fig. 1 A Bland-Altman plot demonstrating the mean differences between LCEA measurements and the $95 \% \mathrm{lim}$ its of agreement.

Fig. 2 A Bland-Altman plot demonstrating the mean differences between AI measurements and the 95\% limits of agreement.
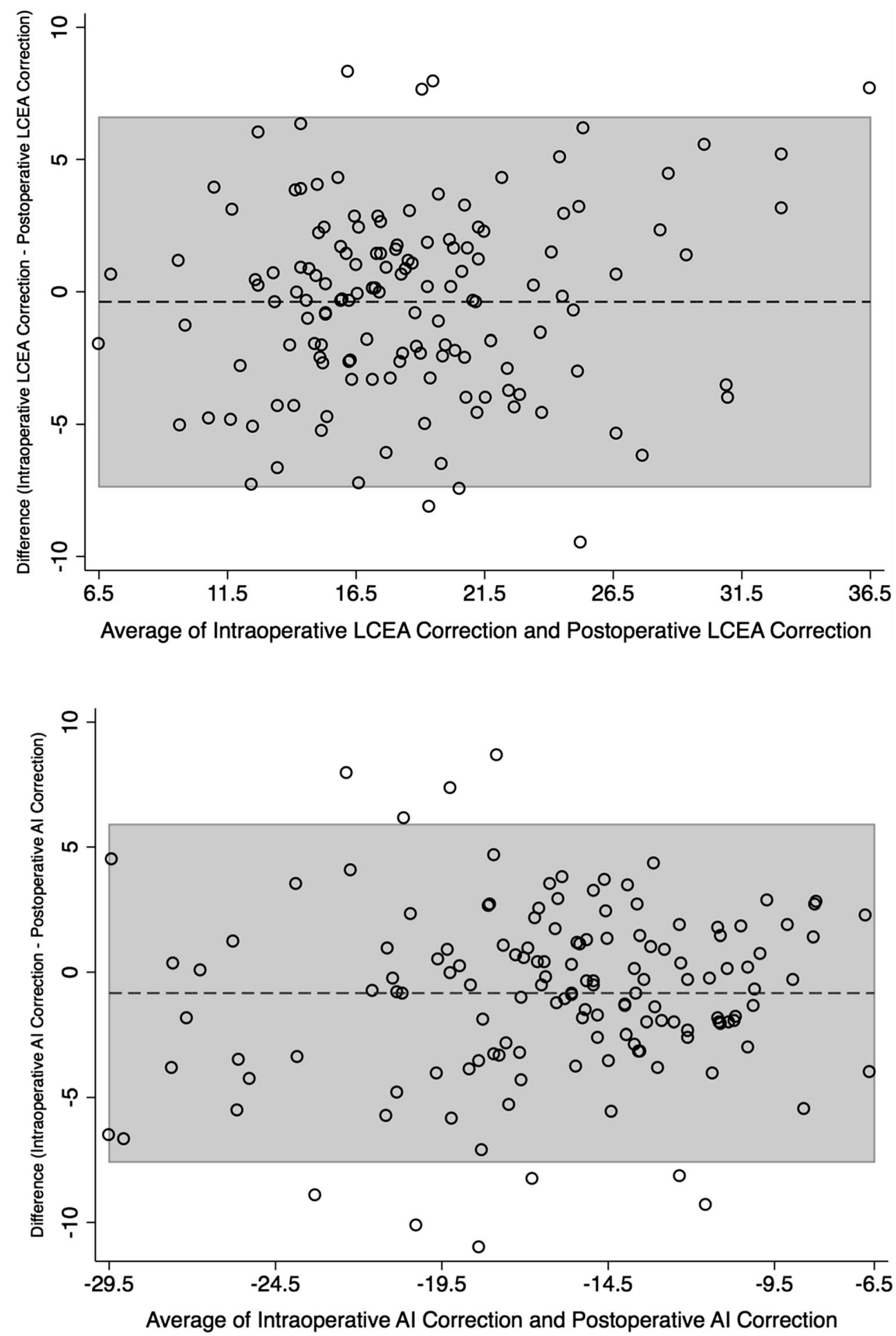

Table 2. Radiographic measurements

\begin{tabular}{llllll}
\hline $\begin{array}{l}\text { Radiographic } \\
\text { measurement }\end{array}$ & $\begin{array}{l}\text { Preoperative } \\
\text { radiograph }\end{array}$ & $\begin{array}{l}\text { Intraoperative } \\
\text { fluoroscopy }\end{array}$ & $\begin{array}{l}\text { Postoperative } \\
\text { radiograph }\end{array}$ & $\begin{array}{l}\text { Intraoperative } \\
\text { correction }\end{array}$ & $\begin{array}{l}\text { Postoperative } \\
\text { correction }\end{array}$ \\
\hline LCEA & $9.7^{\circ}\left(-17^{\circ}\right.$ to $\left.22^{\circ}\right)$ & $28^{\circ}\left(14^{\circ}-39^{\circ}\right)$ & $29^{\circ}\left(15^{\circ}-40^{\circ}\right)$ & $18^{\circ}\left(6^{\circ}-41^{\circ}\right)$ & $19^{\circ}\left(7^{\circ}-33^{\circ}\right)$ \\
Acetabular index & $18^{\circ}\left(6^{\circ}-45^{\circ}\right)$ & $2^{\circ}\left(-7^{\circ}\right.$ to $\left.18^{\circ}\right)$ & $3^{\circ}\left(-7^{\circ}\right.$ to $\left.19^{\circ}\right)$ & $-17^{\circ}\left(-33^{\circ}\right.$ to $\left.6^{\circ}\right)$ & $-16^{\circ}\left(-32^{\circ}\right.$ to $\left.5^{\circ}\right)$ \\
\hline
\end{tabular}

Mean and range for each measurement; LCEA = lateral center-edge angle. 
Table 3. Interrater reliability and agreement between preoperative and postoperative radiographs and intraoperative fluoroscopy (first reads of both observers)

\begin{tabular}{llcccc}
\hline Radiographic measurement & $\mathrm{r}_{\mathrm{c}}$ & $95 \% \mathrm{CI}$ & Bland-Altman average difference (SD) & 95\% limits of agreement & Pearson's $\mathrm{r}$ \\
\hline $\begin{array}{l}\text { Preoperative } \\
\text { LCEA }\end{array}$ & 0.81 & $0.75-0.87$ & $1.17(3.9)$ & -6.5 to 8.9 & -11.2 to 8.5 \\
$\quad$ AI & 0.73 & $0.66-0.81$ & $-1.38(5.0)$ & -7.1 to 5.6 & 0.82 \\
Postoperative & & & & -5.3 to 8.4 & 0.75 \\
$\quad$ LCEA & 0.78 & $0.71-0.84$ & $-0.72(3.2)$ & -6.7 to 5.5 & 0.75 \\
AI & 0.71 & $0.63-0.80$ & $1.6(3.5)$ & -4.8 to 7.6 & 0.78 \\
Fluoroscopy & & & & $-0.57(3.1)$ & 0.74 \\
$\quad$ LCEA & 0.77 & $0.70-0.84$ & $1.4(3.2)$ &
\end{tabular}

$\mathrm{CI}=$ confidence interval; LCEA = lateral center-edge angle; $\mathrm{AI}=$ acetabular index (Tönnis angle).

Table 4. Intrarater reliability and agreement between preoperative and postoperative radiographs and intraoperative fluoroscopy (first and second reads of one observer)

\begin{tabular}{|c|c|c|c|c|c|}
\hline Radiographic measurement & $r_{c}$ & $95 \% \mathrm{CI}$ & $\begin{array}{l}\text { Bland-Altman average } \\
\text { difference (SD) }\end{array}$ & $95 \%$ limits of agreement & Pearson's $r$ \\
\hline \multicolumn{6}{|l|}{ Preoperative } \\
\hline LCEA & 0.85 & $0.81-0.90$ & $-0.6(3.7)$ & -7.8 to 6.5 & 0.86 \\
\hline AI & 0.92 & $0.89-0.94$ & $-0.14(3.04)$ & -6.1 to 5.8 & 0.92 \\
\hline \multicolumn{6}{|l|}{ Postoperative } \\
\hline LCEA & 0.86 & $0.81-0.90$ & $0.09(2.6)$ & -5.0 to 5.2 & 0.87 \\
\hline AI & 0.90 & $0.87-0.93$ & $-0.07(2.31)$ & -4.6 to 4.5 & 0.90 \\
\hline \multicolumn{6}{|l|}{ Fluoroscopy } \\
\hline LCEA & 0.83 & $0.78-0.88$ & $-0.69(2.6)$ & -5.8 to 4.4 & 0.85 \\
\hline $\mathrm{AI}$ & 0.80 & $0.74-0.86$ & $0.51(2.7)$ & -4.7 to 5.7 & 0.81 \\
\hline
\end{tabular}

$\mathrm{CI}=$ confidence interval; LCEA = lateral center-edge angle; $\mathrm{AI}=$ acetabular index (Tönnis angle).

\section{Discussion}

Although the use of fluoroscopy is common in the operating room during orthopaedic procedures, there are relatively few studies reporting the reliability of measures of correction intraoperatively to those found postoperatively on radiographs [3, 10]. Although these studies suggest intraoperative fluoroscopy is a useful tool to judge correction in PAO, both had a small number of subjects and neither reported the intra- or interrater reliability of these measures. The outcome of PAO is dependent on precise reorientation of the acetabular fragment; therefore, intraoperative measures of this reorientation are of paramount importance [6, 15]. Our study demonstrates that fluoroscopy use in the operating room during PAO for acetabular dysplasia demonstrates substantial agreement with postoperative radiographic measures of correction on the AP images and the mean difference between measures is small $\left(<1^{\circ}\right.$, SD 3.6 for LCEA and 3.4 for $\left.\mathrm{AI}\right)$. In addition, we have shown that LCEA and AI have substantial to almost perfect agreement for both intra- and interrater reliability using radiographic and fluoroscopic images, which further validate these measures of acetabular correction intraoperatively.

Our study is not without limitations. This was a retrospective study of our imaging database and therefore is limited by availability of electronic imaging studies. Early in the senior author's practice of PAO (1996-2000), our imaging was completed on radiographic films and consequently these patients had to be excluded from our analysis. In addition, referred patients who did not have electronic images in our PACS system or those whose earlier permanent film had been discarded were also excluded. We also excluded patients with acetabular retroversion and post-Perthes deformity, so our findings are not generalizable to patients with these conditions. Although we believe the included patients are representative of those undergoing PAO at our institution, we acknowledge the possibility of selection bias based on these exclusions. In addition to measures of lateral 
coverage and acetabular inclination, assessment of acetabular reorientation requires the evaluation of anterior coverage and acetabular version. Measures of anterior and posterior coverage are less reliable than the LCEA and AI in cadaveric evaluation [3]. However, Lehmann et al. [10] reported that the ACEA is reliable between intraoperative and postoperative fluoroscopy. We were unable to assess the reliability of the ACEA as a result of the fact that all patients did not have fluoroscopic images of their false profile view saved in the imaging directory. This limited our ability to evaluate the reliability of fluoroscopically assessing anterior acetabular coverage. Therefore, further studies will be needed to determine the reliability of these measures of acetabular version and anterior coverage.

Intraoperative correction can be judged with either intraoperative radiographs or fluoroscopy [7]. Intraoperative radiographs give a larger field of view to view the entire pelvis on one image; however, they require expensive equipment including direct digital capture devices [7]. In addition, the radiation exposure is higher for the surgeon and patient with intraoperative radiographs [8]. Fluoroscopy has lower resolution and a smaller field of view compared with radiographs but is more conveniently used in the operating room. Prior studies have validated fluoroscopy as an imaging modality to judge correction in PAO [3, 8, 10]. Büchler et al. [3] compared fluoroscopy with pelvic radiographs in a cadaver model and found LCEA and AI to be highly correlated between the two imaging modalities. The case series reported have been small studies with a single radiographic reader [8, 10]. Kühnel et al. [8] reported only moderate correlation between intraoperative fluoroscopy and intraoperative radiographs and moderate intrarater reliability, but this may have been the result of their small series of 22 patients. Our findings are similar to Lehmann et al. [10]. Given that AP pelvis radiographs have the $\mathrm{x}$-ray beam centered over the pubic symphysis and fluoroscopic images have the $\mathrm{x}$-ray beam centered over the hip, one may expect this to increase the variation in the AI and LCEA measures between these two imaging modalities. However, we report a high correlation between intraoperative fluoroscopic correction and postoperative radiographic correction with both LCEA and AI in a larger series of patients with multiple readers compared with prior studies.

Prior studies comparing fluoroscopy with intraoperative radiographs or postoperative radiographs have had only single readers and have not reported interrater reliability or agreement $[8,10]$. Multiple prior studies have reported on the reliability of measuring the LCEA and AI on plain radiographs. Studies on the interrater reliability of the LCEA reported intraclass correlation coefficients between 0.73 and $0.95[12,14,16]$. Intrarater reliability for these measures in published studies is between 0.80 and 0.87
[12, 14, 16]. Similarly, moderate to high intra- and interrater reliability is reported for radiographic measures of AI $[12,14,16]$. Given the potential variability in tilt, rotation, and orientation to the pelvis that can take place with fluoroscopy of the hip in the operating room, the importance of this reliability cannot be overstated. It is important to use a standardized protocol to recreate the image of the pelvic ring seen on preoperative radiography or to an appropriate nonrotated AP of the pelvic ring before obtaining images of the hip with the fluoroscope. Lehmann et al. [10] reported using similar methods in their study, which likely accounts for the similar findings validating intraoperative fluoroscopy in the two studies.

Our work further validates intraoperative fluoroscopy as an accurate and reliable tool to judge intraoperative correction of the acetabular fragment during PAO for symptomatic acetabular dysplasia. Further areas for study in this regard include applying similar methodology to the correction of anterior coverage of the acetabulum and acetabular version with the use of intraoperative fluoroscopy. Finally, the use of fluoroscopy in the correction of acetabular retroversion or acetabular reorientation in the setting of a post-Perthes deformity also merits further study.

\section{References}

1. Bland JM, Altman DG. Statistical methods for assessing agreement between two methods of clinical measurement. Lancet. 1986;1:307-310

2. Bland JM, Altman DG. Applying the right statistics: analyses of measurement studies. Ultrasound Obstet Gynecol. 2003;22:8593.

3. Büchler L, Schwab JM, Whitlock PW, Beck M, Tannast M. Intraoperative evaluation of acetabular morphology in hip arthroscopy comparing standard radiography versus fluoroscopy: a cadaver study. Arthroscopy. 2016;32:1030-1037.

4. Clohisy JC, Carlisle JC, Beaulé PE, Kim Y-J, Trousdale RT, Sierra RJ, Leunig M, Schoenecker PL, Millis MB. A systematic approach to the plain radiographic evaluation of the young adult hip. J Bone Joint Surg Am. 2008;90(Suppl 4):47-66.

5. Gala L, Clohisy JC, Beaule PE. Hip dysplasia in the young adult. J Bone Joint Surg Am. 2016;98:63-73.

6. Hartig-Andreasen C, Troelsen A, Thillemann TM, Søballe K. What factors predict failure 4 to 12 years after periacetabular osteotomy? Clin Orthop Relat Res. 2012;470:2978-2987.

7. Kosuge D, Cordier T, Solomon LB, Howie DW. Dilemmas in imaging for peri-acetabular osteotomy: the influence of patient position and imaging technique on the radiological features of hip dysplasia. Bone Joint J. 2014;96:1155-1160.

8. Kühnel SP, Kalberer FA, Dora CF. Periacetabular osteotomy: validation of intraoperative fluoroscopic monitoring of acetabular orientation. Hip Int. 2011;21:303-310.

9. Landis JR, Koch GG. The measurement of observer agreement for categorical data. Biometrics. 1977;33:159-174.

10. Lehmann CL, Nepple JJ, Baca G, Schoenecker PL, Clohisy JC. Do fluoroscopy and postoperative radiographs correlate for 
periacetabular osteotomy corrections? Clin Orthop Relat Res. 2012;470:3508-3514.

11. Lin LI. A concordance correlation coefficient to evaluate reproducibility. Biometrics. 1989;45:255-268.

12. Mast NH, Impellizzeri F, Keller S, Leunig M. Reliability and agreement of measures used in radiographic evaluation of the adult hip. Clin Orthop Relat Res. 2010;469:188-199.

13. Müller R, Büttner P. A critical discussion of intraclass correlation coefficients. Stat Med. 1994;13:2465-2476.

14. Nelitz M, Guenther KP, Gunkel S, Puhl W. Reliability of radiological measurements in the assessment of hip dysplasia in adults. Br J Radiol. 1999;72:331-334.
15. Siebenrock KA, Schöll E, Lottenbach M, Ganz R. Bernese periacetabular osteotomy. Clin Orthop Relat Res. 1999;363:9-20.

16. Tannast M, Mistry S, Steppacher SD, Reichenbach S, Langlotz F, Siebenrock KA, Zheng G. Radiographic analysis of femoroacetabular impingement with Hip2Norm-reliable and validated. $J$ Orthop Res. 2008;26:1199-1205.

17. Tibor LM, Sink EL. Periacetabular osteotomy for hip preservation. Orthop Clin North Am. 2012;43:343-357.

18. Tönnis D. Normal values of the hip joint for the evaluation of $\mathrm{x}$-rays in children and adults. Clin Orthop Relat Res. 1976;119:39-47.

19. Wiberg G. The anatomy and roentgenographic appearance of a normal hip joint. Acta Chir Scand. 1939;7-38. 